\title{
Calculation of two-center overlap integral in molecular coordinate system over Slater type orbital using Löwdin $\alpha$-radial and Guseinov rotation-angular functions
}

\author{
B. A. Mamedov • H. Koç
}

Received: 29 July 2008 / Accepted: 5 August 2008 / Published online: 5 September 2008

(C) Springer Science+Business Media, LLC 2008

Erratum to: J Math Chem (2008) 44:365

DOI 10.1007/s10910-007-9313-z

The authors regret that in the above article Equations (6) and (7) were some misprints. They are now reproduced correctly below:

$$
\begin{aligned}
S_{n l \lambda, n^{\prime} l^{\prime} \lambda}\left(\zeta, \zeta^{\prime}, R\right)= & N_{n l, n^{\prime} l^{\prime} \lambda}(-1)^{l^{\prime}+\lambda}\left(\frac{\zeta}{\zeta^{\prime}}\right)^{n+\frac{1}{2}} \\
& \times \sum_{i=0}^{n^{\prime}+l^{\prime}+l} \sum_{j=0}^{n^{\prime}+l}(n-l+j) ! C_{l}^{n^{\prime} l^{\prime} \lambda}(i, j)\left(\zeta^{\prime} R\right)^{n-2 l-l^{\prime}+i+j} \\
& \times\left[e ^ { - \zeta ^ { \prime } R } \left(\frac{(-1)^{j}}{\left.\left[R\left(\zeta-\zeta^{\prime}\right)\right]^{n-l+j+1}-\frac{1}{\left[R\left(\zeta+\zeta^{\prime}\right)\right]^{n-l+j+1}}\right)}\right.\right. \\
& \left.+e^{-\zeta R} \sum_{k=0}^{n-l+j} \frac{1}{(n-l+j-k) !}\left(\frac{(-1)^{i}}{\left[R\left(\zeta+\zeta^{\prime}\right)\right]^{k+1}}-\frac{(-1)^{j}}{\left[R\left(\zeta-\zeta^{\prime}\right)\right]^{k+1}}\right)\right] \\
& \text { for } \left.\zeta \neq \zeta^{\prime}\right) \\
N_{n l, n^{\prime} l^{\prime} \lambda=} & (-1)^{\lambda} 2^{n^{\prime}+n}\left[\frac{(2 l+1)\left(2 l^{\prime}+1\right)\left(l^{\prime}+\lambda\right) !(l-\lambda) !}{(2 n) !\left(2 n^{\prime}\right) !(l+\lambda) !\left(l^{\prime}-\lambda\right) !}\right]^{\frac{1}{2}}
\end{aligned}
$$

The online version of the original article can be found under doi:10.1007/s10910-007-9313-z.

B. A. Mamedov $(\bowtie) \cdot$ H. Koç

Department of Physics, Faculty of Arts and Sciences, Gaziosmanpaşa University, Tokat, Turkey e-mail: bamamedov@yahoo.com 